\title{
Synthesis, Characterization and Biological Efficacies from Some New Dinuclear Metal Complexes for Base 3-(3,4-Dihydroxy-phenyl)-2-[(2-hydroxy-3-methylperoxy- benzylidene)-amino]-2-methyl Propionic Acid
}

\author{
Shatha Mohammed Hassan Obaid ${ }^{1}$, Jasim Shihab Sultan ${ }^{1}$, Abbas Ali Salih Al-Hamdani2 ${ }^{2, *}$ \\ ${ }^{1}$ Department of Chemistry, College of Education for Pure Science - Ibn Al-Haitham, University of Baghdad, Iraq \\ ${ }^{2}$ Department of Chemistry, College of Science for Women, University of Baghdad, Baghdad, Iraq
}

\section{*Corresponding author:}

email: Abbas_alhamadani@yahoo.co.uk

Received: September 20, 2019

Accepted: January 20, 2020

DOI: $10.22146 / \mathrm{ijc} .49842$
Abstract: The reaction of methyldopa with o-vanillin in refluxing ethanol afforded Schiff base and characterized through physical analysis with a number of spectra also the study of biological activity. The geometry of the Schiff base was identified through using (C.H.N) analysis, Mass, ${ }^{1} H$-NMR, FT-IR, UV-Vis spectroscopy. Metal complexes of $\mathrm{Cr}^{3+}, \mathrm{Mn}^{2+}, \mathrm{Co}^{2+}, \mathrm{Ni}^{2+}, \mathrm{Cu}^{2+}, \mathrm{Zn}^{2+}, \mathrm{Cd}^{2+}$ and $\mathrm{Hg}^{2+}$ with Schiff base have been prepared in the molar ratio 2:1 (Metal:L), ( $L=$ Schiff base ligand) except $\mathrm{Hg}^{2+}$ at molar ratio 1:1 $(H g: L)$. The prepared complexes were characterized by using Mass, FT-IR and UV-Vis spectral studies, on other than magnetic properties and flame atomic absorption, conductivity measurements. According to the results a dinuclear octahedral geometry has been suggested for $\mathrm{Cr}^{3+}, \mathrm{Mn}^{2+}, \mathrm{Co}^{2+}, \mathrm{Ni}^{2+}, \mathrm{Cu}^{2+}$ and $\mathrm{Zn}^{2+}$ complexes, dinuclear tetrahedral for $\mathrm{Cd}^{2+}$ and mononuclear tetrahedral for $\mathrm{Hg}^{2+}$ complex. This work highlights the relevance of metal complexation strategy to stabilize the ligands and improve their bioactivity. Schiff base complexes have been screen for their antibacterial activity against Gram negative and positive bacteria and antifungal activity showing promising antibacterial and biological activity.

Keywords: methyldopa; o-vanillin; Schiff base complexes; spectral studies; biological efficacy

\section{- INTRODUCTION}

Schiff bases a special class of organic ligands with a variety of donor atoms revealing interesting coordination modes towards numerous metals [1-2]. These are the condensation product of an active carbonyl group acting as an electrophile and an amino group as a nucleophile. Due to their structural varieties and unique characteristics, these are the most versatile studied ligands in coordination chemistry. Among the Schiff base derivatives, those having phenol moiety have attracted considerable attention due to their wide range of biological activities Metal complexes such as $\mathrm{Zn}, \mathrm{Cd}$, Co, $\mathrm{Ni}, \mathrm{Pd}, \mathrm{Ag}$, and $\mathrm{Hg}$ have different biological activities such as antimicrobial [3-4], antifungal [5-7], antioxidant [5,8] and anticancer [9-12]. These type complexes have also many different properties such as catalytic activity, porosity, magnetism, and conductivity [13-14]. C-C and $\mathrm{C}-\mathrm{N}$ cross-coupling reactions are very important for natural products, drug design and industrial starting materials. For this reason, it is an important reaction group for organic chemistry. Among the Schiff base derivatives, those having carbonyl and phenol groups' moiety have attracted considerable attention due to their wide range of biological activities [15-16]. Methyldopa (M-dopa), the L-isomer of alpha-methyldopa, is levo-3(3,4-dihydroxyphenyl)-2-methylalanine. Its empirical methyldopa is used in the clinical treatment of the following disorders: hypertension (or high blood pressure) gestational hypertension (or pregnancyinduced hypertension). Aldomet (L-methyldopa) is an antihypertensive drug and is an aromatic amino-acid de carboxyl's inhibitor in animals and man. A literature survey revealed that a little work has been reported on 
metal complexes of Schiff bases derived from methyldopa derivative [17], Schiff bases derived from $o$-vanillin are well known for their interesting ligational properties and exclusive applications in different fields. The interaction of these donor ligands and metal ions gives complexes of different geometries, and a literature survey reveals that these complexes are potentially more biologically active. Thus, in recent years Schiff bases and their metal complexes have attained much attraction because of their extensive biological activities [18-19]. The authors reported the catalytic effects of the Schiff base. The present paper aims to prepare, characterize the chemical structure and to study the antibacterial activity and antifungal from Schiff base ligand prepared which is derived from methyldopa as well as $o$-vanillin and its metal complexes with $\mathrm{Cr}^{3+}, \mathrm{Mn}^{2+}, \mathrm{Co}^{2+}, \mathrm{Ni}^{2+}, \mathrm{Cu}^{2+}, \mathrm{Zn}^{2+}$, $\mathrm{Cd}^{2+}$ and $\mathrm{Hg}^{2+}$ are being reported.

\section{- EXPERIMENTAL SECTION}

\section{Materials}

All the reagents used for the synthesis of methyldopa (Sigma-Aldrich), $o$-vanillin (Fluka), solvents such as aceton, chloroform as well benzene (Merck), but dimethyl sulfoxide also dimethylformamide $(\mathrm{BDH})$, and inorganic salts such as $\mathrm{CrCl}_{3} \cdot 6 \mathrm{H}_{2} \mathrm{O}, \mathrm{MnCl}_{2} \cdot 4 \mathrm{H}_{2} \mathrm{O}, \mathrm{NiCl}_{2} \cdot 6 \mathrm{H}_{2} \mathrm{O}$, $\mathrm{CuCl}_{2} \cdot \mathrm{H}_{2} \mathrm{O}$ also $\mathrm{HgCl}_{2}$ (Merck), $\mathrm{CoCl}_{2} \cdot 6 \mathrm{H}_{2} \mathrm{O}$ (RiedialDehaen), $\mathrm{ZnCl}_{2}$ (Aldrih) as well as $\mathrm{CdCl}_{2} \cdot \mathrm{H}_{2} \mathrm{O}$ (Fluka) and were used as supplied.

\section{Instrumentation}

Melting points of the Schiff base ligand and all its metal complexes were determined by using Stuart Melting Point Apparatus. Metal contents to all compounds have been determined by using atomic absorption technicality by AA- 680 Shimadzu. The conductometric measurements of the Schiff base ligand and metal complexes were carried out in DMSO solution using CON 510 Conductivity at room temperature. Vibrational spectra were recorded to the Schiff base ligand and all complexes using $\mathrm{KBr}$ pellets on FT-IR-600 FT-IR Spectrophotometer, in the region $4000-400 \mathrm{~cm}^{-1}$ range. ${ }^{1} \mathrm{H}-\mathrm{NMR}$ for Schiff base ligand was registered on by NMR Bruker $400 \mathrm{MHz}$ at DMSO- $\mathrm{d}_{6}$ for TMS as the inner standard. Mass spectra to Schiff base ligand as well all its compounds have been registered by MS Model 5973 Network Mass Selection Technology (HP) with Triple-Axis Detector by the analyzer Quadrupole at $230^{\circ} \mathrm{C}$. Electronic spectral studies were performed on using Shimadzu-U.V-160 to the Schiff base ligand and its metal complexes as well as metal(II) complexes at DMSO $\left(10^{-3} \mathrm{M}\right)$ in the range (2001100) $\mathrm{nm}$. Magnetic susceptibility for prepared metal(II) complexes was measured on Auto Magnetic Susceptibility Balance Sherwood Scientific. In the chloride test with each complex $\left(5 \times 10^{-2} \mathrm{~g}\right)$ has been washed using concentrated nitric acid and diluted with water. To the resultant solution from the complexes, an aqueous solution of silver nitrate was added, a white precipitate of silver chloride was formed in the case of metals complexes has chloride content where as in the case of metals complexes [20], no precipitate was observed with all metal complexes.

\section{Procedure}

Prepare for Schiff base ligand [3-(3,4-dihydroxyphenyl)-2-[(2-hydroxy-3-methylperoxy-benzylidene)amino]-2-methyl propionic acid]

Schiff base ligand (L) has been prepared through adding $1 \mathrm{mmol}$ of $(0.2112 \mathrm{~g})$ of methyldopa (2-amino-3(3,4-dihydroxy-phenyl)-2-methyl-propionic acid) with $25 \mathrm{~mL}$ ) of ethanol with ( $1 \mathrm{mmol}$ of $0.0040 \mathrm{~g}$ ) of sodium hydroxide to the solution and was added $(1 \mathrm{mmol}$, $0.1522 \mathrm{~g}$ ) of $o$-vanillin (2-hydroxy-3-methoxybenzaldehyde) dissolved of $(25 \mathrm{~mL})$ ethanol. The mixture was refluxed with stirring for $(7 \mathrm{~h})$. The resulting solution was evaporated to half volume and the precipitated produced was collected by filtration, washed twice with distilled water, and dried over anhydrous calcium chloride. The deep orange solid mass formed. Yield: 70\%. \%Calculated: C: 62.599\%, H: 5.545\%, N: 4.056\%. Found: C: $61.511 \%, \quad H: 4.056 \%$, N: $3.609 \%$. The condensation of methyldopa and $o$-vanillin in ethanol gives according to the following reaction in Scheme 1.

\section{Preparation of the metal complexes}

A general method has been used for prepare of all chelate complexes except the $\mathrm{Hg}^{2+}$ complex. A solution $(0.3454 \mathrm{~g}, 1 \mathrm{mmol})$ of Schiff base ligand dissolved in $(20 \mathrm{~mL})$ of ethanol with $(0.0040 \mathrm{~g}, 1 \mathrm{mmol})$ of sodium 


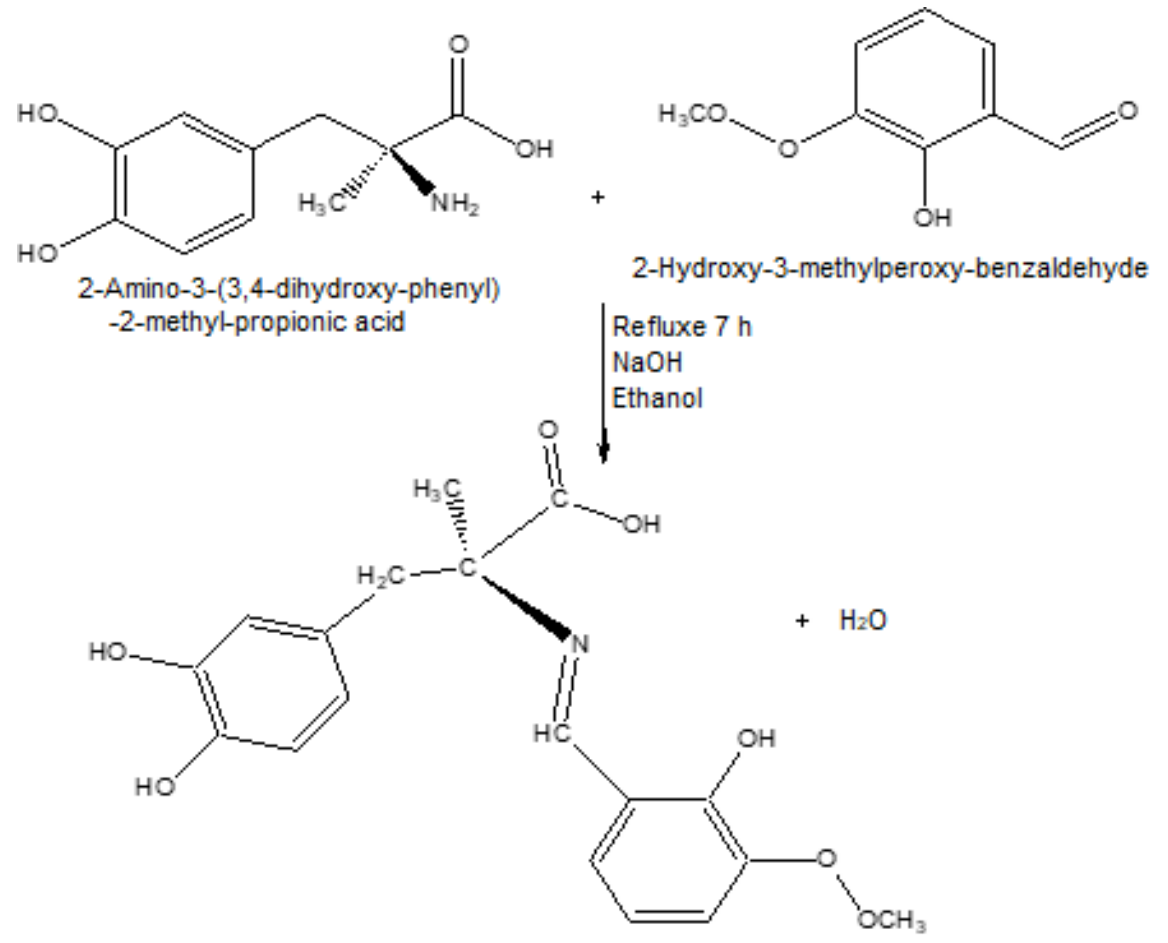

3-(3,4-Dihydroxy-phenyl)-2[(2-hydroxy-3-methylperoxy-benzylidene) -amino]-2-methyl-propionic acid

Scheme 1. Synthesis of Schiff base ligand (L)

hydroxide was added with stirring a stoichiometric amount (1:2) (ligand:metal) ratio for $\mathrm{CrCl}_{3} \cdot 6 \mathrm{H}_{2} \mathrm{O}(0.4529 \mathrm{~g}$, $2 \mathrm{mmol}), \mathrm{MnCl}_{2} \cdot 4 \mathrm{H}_{2} \mathrm{O}(0.394 \mathrm{~g}, 2 \mathrm{mmol}), \mathrm{CoCl}_{2} \cdot 6 \mathrm{H}_{2} \mathrm{O}$ (0.476 g, $2 \mathrm{mmol}), \mathrm{NiCl}_{2} \cdot 6 \mathrm{H}_{2} \mathrm{O}(0.476 \mathrm{~g}, 2 \mathrm{mmol})$, $\mathrm{CuCl}_{2} \cdot 2 \mathrm{H}_{2} \mathrm{O}(0.3410 \mathrm{~g}, 2 \mathrm{mmol}), \mathrm{ZnCl}_{2}(0.372 \mathrm{~g}, 2 \mathrm{mmol})$ and $\mathrm{CdCl}_{2} \cdot \mathrm{H}_{2} \mathrm{O}(0.402 \mathrm{~g}, 2 \mathrm{mmol})$. But the $\mathrm{Hg}^{2+}$ complex with a stoichiometric amount (1:1) (ligand:metal) ratio for $\mathrm{HgCl}_{2}(0.2715 \mathrm{~g}, 1 \mathrm{mmol})$, dissolved with distilled water. Each mixture was refluxed for $3 \mathrm{~h}$ for each of the eight complexes. At room temperature on cooling, colored compounds deposited out in every case. They have been filtrated, washed for acetone as well as dried over anhydrous calcium chloride.

\section{Biological activities}

The biological activities of Schiff base ligand and its metal complexes have been studied for their antibacterial against four species of bacteria two strain from a gram positive as well two strain of a gram negative and antifungal against Candida albicans by diffusion method [21]. The screened compounds were dissolved individually in DMSO in order to make up a solution of
$10^{-3} \mathrm{M}$ concentration for each from these complexes. Discs have been placed at the exterior from agar solid nutrients dishes seeded through the examined bacteria and fungi. Diameters of inhibition zones $(\mathrm{mm})$ have been measured on the end from a nursery period that was $24 \mathrm{~h}$ at $37^{\circ} \mathrm{C}$ with bacteria and fungi.

\section{- RESULTS AND DISCUSSION}

\section{Elemental Analysis and Conductance Measurements}

The reaction of the Schiff base ligand with the metal chloride $\left(\mathrm{Cr}^{3+}, \mathrm{Mn}^{2+}, \mathrm{Co}^{2+}, \mathrm{Ni}^{2+}, \mathrm{Cu}^{2+}, \mathrm{Zn}^{2+}, \mathrm{Cd}^{2+}\right.$ as well $\mathrm{Hg}^{2+}$ ) gave colored complexes at good yields. The analytical data along with some physical properties of the ligand and its metal complexes are summarized in Table 1. The Schiff base ligand, on interaction with $\mathrm{Cr}^{3+}$, $\mathrm{Mn}^{2+}, \mathrm{Co}^{2+}, \mathrm{Ni}^{2+}, \mathrm{Cu}^{2+}, \mathrm{Zn}^{2+}, \mathrm{Cd}^{2+}$, and $\mathrm{Hg}^{2+}$, yields compounds corresponding to the general formula $\left.\left[\mathrm{M}_{2}(\mathrm{~L})\left(\mathrm{H}_{2} \mathrm{O}\right)_{\mathrm{n}}\right] \mathrm{X}_{2} \cdot \mathrm{H}_{2} \mathrm{O} \cdot\left[\mathrm{M}=\mathrm{Cr}^{3+}, \mathrm{n}=7, \mathrm{X}=2\right)\right],[\mathrm{M}=$ $\left.\left(\mathrm{Mn}^{2+}, \mathrm{Co}^{2+}, \mathrm{Ni}^{2+}, \mathrm{Cu}^{2+}, \mathrm{Zn}^{2+}\right), \mathrm{n}=7, \mathrm{X}=0\right],\left[\mathrm{M}=\mathrm{Cd}^{2+}, \mathrm{n}\right.$ $=3, \mathrm{X}=0]$ and $\left[\mathrm{M}=\mathrm{Hg}^{2+}, \mathrm{n}=1, \mathrm{X}=0\right.$ ] Fig. 1. The analytical data show that the metal to ligand ratio of the 

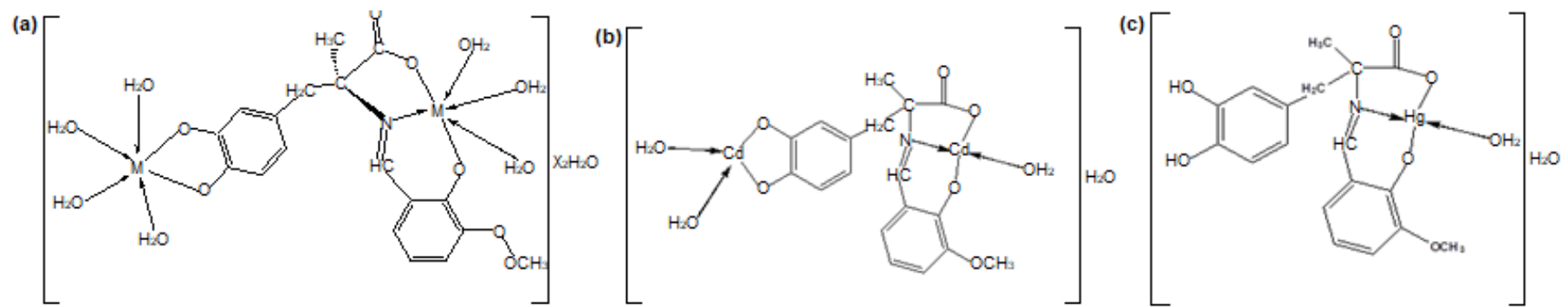

Fig 1. (a) Binuclear octahedral for $\mathrm{Cr}^{3+}, \mathrm{Mn}^{2+}, \mathrm{Co}^{2+}, \mathrm{Ni}^{2+}, \mathrm{Cu}^{2+}$ and $\mathrm{Zn}^{2+}$ complexes, (b) Binuclear tetrahedral for $\mathrm{Cd}^{2+}$ complex and (c) Mononuclear tetrahedral for $\mathrm{Hg}^{2+}$ complex

Table 1. Analytical and physical data for Schiff base ligand as well as its compounds

\begin{tabular}{|c|c|c|c|c|c|c|}
\hline Compounds & MWt./g mol ${ }^{-1}$ & $\begin{array}{l}\text { Empirical } \\
\text { Formula }\end{array}$ & Color & $\mathrm{MP}^{\circ} \mathrm{C}$ & $\begin{array}{l}\text { M\% Calculate } \\
\text { (Found) }\end{array}$ & $\begin{array}{l}\Lambda_{\mathrm{m}}\left(\mathrm{ohm}^{-1} \mathrm{~cm}^{2} \mathrm{~mol}^{-1}\right) \\
\text { in DMSO, } 10^{-3} \mathrm{M} .\end{array}$ \\
\hline Schiff base ligand (L) & 345.37 & $\mathrm{C}_{18} \mathrm{H}_{19} \mathrm{NO}_{6}$ & Deep orange & 120 & - & - \\
\hline$\left[\mathrm{Cr}_{2}(\mathrm{~L})\left(\mathrm{H}_{2} \mathrm{O}\right)_{7}\right] \cdot \mathrm{Cl}_{2} \mathrm{H}_{2} \mathrm{O}$ & 660.27 & $\mathrm{C}_{18} \mathrm{H}_{31} \mathrm{NO}_{14} \mathrm{Cl}_{2} \mathrm{Cr}_{2}$ & Deep green & $\begin{array}{l}>350 \\
\text { dec. }\end{array}$ & $\begin{array}{l}15.75 \\
(14.17)\end{array}$ & 52.30 \\
\hline $\begin{array}{l}{\left[\mathrm{Mn}_{2}(\mathrm{~L})\left(\mathrm{H}_{2} \mathrm{O}\right)_{7}\right] \cdot \mathrm{H}_{2} \mathrm{O}} \\
\text { Valine }(\mathrm{Val})\end{array}$ & 595.33 & $\mathrm{C}_{18} \mathrm{H}_{31} \mathrm{NO}_{14} \mathrm{Mn}_{2}$ & Deep green & $\begin{array}{l}>350 \\
\text { dec. }\end{array}$ & $\begin{array}{l}18.46 \\
(17.67)\end{array}$ & 25.64 \\
\hline $\begin{array}{l}{\left[\mathrm{Co}_{2}(\mathrm{~L})\left(\mathrm{H}_{2} \mathrm{O}\right)_{7}\right] \cdot \mathrm{H}_{2} \mathrm{O}} \\
{\left[\mathrm{Co}(\mathrm{Ala})(\mathrm{Val})\left(\mathrm{H}_{2} \mathrm{O}\right)_{2}\right] \mathrm{H}_{2}}\end{array}$ & 603.23 & $\mathrm{C}_{18} \mathrm{H}_{31} \mathrm{NO}_{14} \mathrm{Co}_{2}$ & Deep brown & $\begin{array}{l}>350 \\
\text { dec. }\end{array}$ & $\begin{array}{l}19.54 \\
(18.92)\end{array}$ & 27.14 \\
\hline$\left[\mathrm{Ni}_{2}(\mathrm{~L})\left(\mathrm{H}_{2} \mathrm{O}\right)_{7}\right] \cdot \mathrm{H}_{2} \mathrm{O}$ & 602.75 & $\mathrm{C}_{18} \mathrm{H}_{31} \mathrm{NO}_{14} \mathrm{Ni}_{2}$ & Deep brown & $\begin{array}{l}>350 \\
\text { dec. }\end{array}$ & $\begin{array}{l}19.48 \\
(18.19)\end{array}$ & 28.94 \\
\hline$\left[\mathrm{Cu}_{2}(\mathrm{~L})\left(\mathrm{H}_{2} \mathrm{O}\right)_{7}\right] \cdot \mathrm{H}_{2} \mathrm{O}$ & 612.46 & $\mathrm{C}_{18} \mathrm{H}_{31} \mathrm{NO}_{14} \mathrm{Cu}_{2}$ & Deep brown & $\begin{array}{l}>350 \\
\text { dec. }\end{array}$ & $\begin{array}{l}20.75 \\
(19.01)\end{array}$ & 27.14 \\
\hline$\left[\mathrm{Zn}_{2}(\mathrm{~L})\left(\mathrm{H}_{2} \mathrm{O}\right)_{7}\right] \cdot \mathrm{H}_{2} \mathrm{O}$ & 616.13 & $\mathrm{C}_{18} \mathrm{H}_{31} \mathrm{NO}_{14} \mathrm{Zn}_{2}$ & Pale green & $\begin{array}{l}>350 \\
\text { dec. }\end{array}$ & $\begin{array}{l}21.22 \\
(21.06)\end{array}$ & 23.44 \\
\hline$\left[\mathrm{Cd}_{2}(\mathrm{~L})\left(\mathrm{H}_{2} \mathrm{O}\right)_{3}\right] \cdot \mathrm{H}_{2} \mathrm{O}$ & 638.19 & $\mathrm{C}_{18} \mathrm{H}_{25} \mathrm{NO}_{10} \mathrm{Cd}_{2}$ & Pale green & $\begin{array}{l}>350 \\
\text { dec. }\end{array}$ & $\begin{array}{l}35.23 \\
(34.48)\end{array}$ & 24.04 \\
\hline$\left[\mathrm{Hg}(\mathrm{L})\left(\mathrm{H}_{2} \mathrm{O}\right)\right] \cdot \mathrm{H}_{2} \mathrm{O}$ & 579.96 & $\mathrm{C}_{18} \mathrm{H}_{21} \mathrm{NO}_{8} \mathrm{Hg}$ & Pale brown & $\begin{array}{l}>350 \\
\text { dec. }\end{array}$ & $\begin{array}{l}34.59 \\
(33.73)\end{array}$ & 25.94 \\
\hline
\end{tabular}

dec. = Decompose

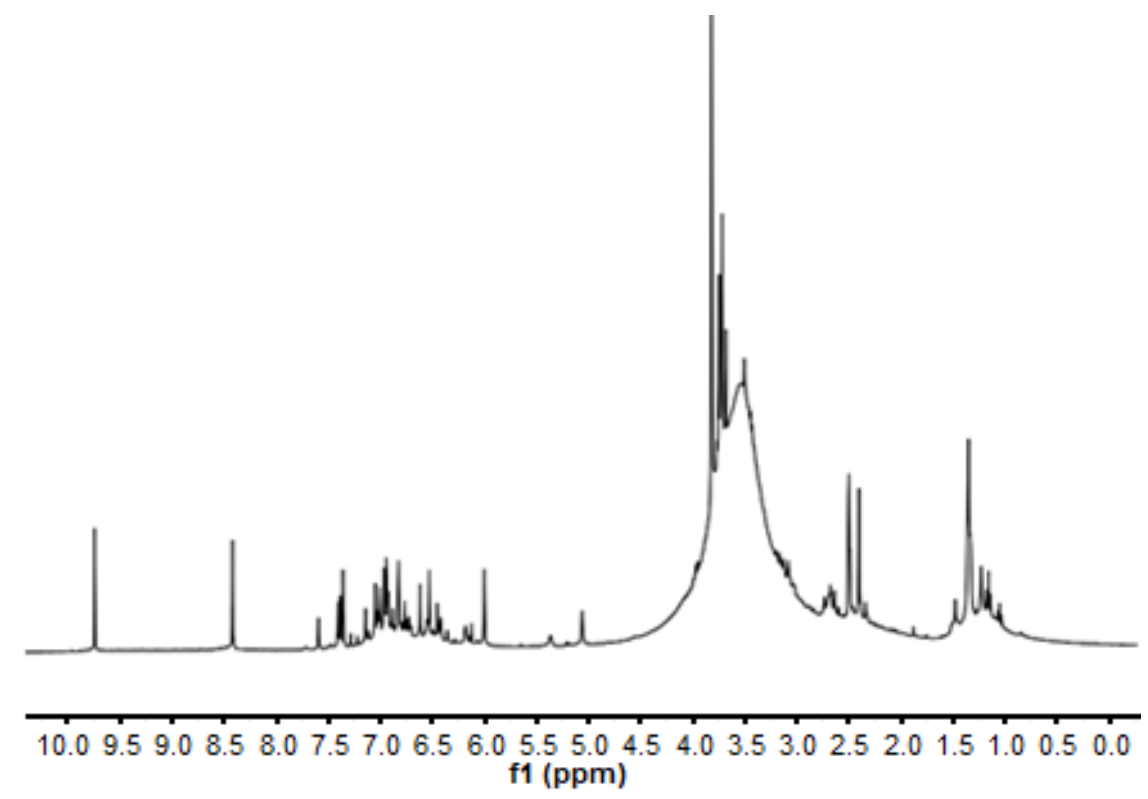

Fig 2. ${ }^{1} \mathrm{H}-\mathrm{NMR}$ spectrum for Schiff base ligand 
complexes is (2:1) except the $\mathrm{Hg}^{2+}$ complex is (1:1). The low molar conductance values of the complexes except the $\mathrm{Cr}^{3+}$ complex reveal their non-electrolytic nature [22]. All the complexes are no hygroscopic, stable, solid, stable in water except $\mathrm{Cr}^{3+}$ complex and at common organic solvents like acetone, chloroform, and benzene, but soluble at ethanol, methanol, DMSO and DMF.

\section{${ }^{1} \mathrm{H}-N M R$ Spectra of the Schiff Base Ligand}

${ }^{1} \mathrm{H}-\mathrm{NMR}$ spectra for Schiff base ligand (L) at DMSO- $\mathrm{d}_{6}$ solvent in Fig. 2, appears chemical shift at $\delta=$ 9.74 and $8.42 \mathrm{ppm}$ due to $-\mathrm{OH}$ proton of the carboxyl and azomethane groups respectively. The spectrum displays various signals in $\delta=6.00-7.58 \mathrm{ppm}$ assigned into aromatic protons, the signals at $\delta=5.21,5.25$ and $5.45 \mathrm{ppm}$ lead to $(-\mathrm{OH})$ of phenol. Resonance in $\delta=$ $2.73,1.18$, and $3.82 \mathrm{ppm}$ due to protons of $\mathrm{CH}_{2}, \mathrm{CH}_{3}$ and methoxy groups sequences, the signals at $\delta=2.50 \mathrm{ppm}$ and $\delta=3.50$ indicated into DMSO- $\mathrm{d}_{6}$ and water $\left(\mathrm{H}_{2} \mathrm{O}\right)$ respectively [23-25].

\section{UV-Visible Spectra and Magnetic Susceptibility Measurements}

UV-Vis spectra for Schiff base ligand as well as its metal compounds dissolved at DMSO $\left(10^{-3} \mathrm{M}\right)$ have been obtained and listed in Table 2. The Schiff base ligand

Table 2. Electronic spectral of the Schiff base ligand and its metal complexes

\begin{tabular}{|c|c|c|c|c|c|c|c|}
\hline Compounds & $(\lambda \mathrm{nm})$ & ABS & w.n $\mathrm{cm}^{-1}$ & $\begin{array}{l}\Theta_{\max } \\
\left(\mathrm{L} \mathrm{mol}^{1} \mathrm{~cm}^{-1}\right)\end{array}$ & Assignments & $\mu_{\text {eff }}($ B.M.) & $\begin{array}{l}\text { Suggested } \\
\text { Structure }\end{array}$ \\
\hline \multirow[t]{2}{*}{ Ligand } & 279 & 1.019 & 35842 & 1019 & $\pi-\pi^{*}$ & - & - \\
\hline & 342 & 0.486 & 29239 & 486 & $\mathrm{n}-\pi^{*}$ & & \\
\hline \multirow[t]{5}{*}[\mathrm{Cr}_{2}(\mathrm{L})(\mathrm{H}_{2}\mathrm{O})_{7}]{$\cdot \mathrm{Cl}_{2} \cdot \mathrm{H}_{2} \mathrm{O}$} & 276 & 2.202 & 36231 & 2202 & L.F. & 3.66 & Octahedral \\
\hline & 364 & 0.947 & 27472 & 947 & C.T. & & \\
\hline & 449 & 0.634 & 22271 & 634 & ${ }^{4} \mathrm{~A}_{2} \mathrm{~g} \rightarrow{ }^{4} \mathrm{~T}_{1} \mathrm{~g}(\mathrm{p})$ & & \\
\hline & 552 & 0.036 & 18115 & 36 & ${ }^{4} \mathrm{~A}_{2} \mathrm{~g} \rightarrow{ }^{4} \mathrm{~T}_{1} \mathrm{~g}(\mathrm{~F})$ & & \\
\hline & 670 & 0.028 & 14925 & 28 & ${ }^{4} \mathrm{~A}_{2} \mathrm{~g} \rightarrow{ }^{4} \mathrm{~T}_{2} \mathrm{~g}(\mathrm{~F})$ & & \\
\hline \multirow[t]{5}{*}[\mathrm{Mn}_{2}(\mathrm{L})(\mathrm{H}_{2}\mathrm{O})_{7}]{$\cdot \mathrm{H}_{2} \mathrm{O}$} & 277 & 1.509 & 36101 & 1509 & L.F. & 6.05 & Octahedral \\
\hline & 347 & 0.777 & 28818 & 777 & C.T. & & \\
\hline & 380 & 0.566 & 26315 & 566 & C.T. & & \\
\hline & 520 & 0.203 & 19230 & 203 & ${ }^{6} \mathrm{~A}_{1} \mathrm{~g} \rightarrow{ }^{4} \mathrm{~A}_{1} \mathrm{~g},{ }^{4} \mathrm{Eg}(\mathrm{G})$ & & \\
\hline & 809 & 0.027 & 12360 & 27 & $\begin{array}{l}{ }^{6} \mathrm{~A}_{1} \mathrm{~g} \rightarrow{ }^{4} \mathrm{~T}_{2} \mathrm{~g}(\mathrm{G}) \\
{ }^{6} \mathrm{~A}_{1} \mathrm{~g} \rightarrow{ }^{4} \mathrm{~T}_{1} \mathrm{~g}(\mathrm{G})\end{array}$ & & \\
\hline \multirow[t]{6}{*}[\mathrm{Co}_{2}(\mathrm{L})(\mathrm{H}_{2}\mathrm{O})_{7}]{$\cdot \mathrm{H}_{2} \mathrm{O}$} & 276 & 2.347 & 36231 & 2347 & $\mathrm{~L}-\mathrm{F}$ & 5.25 & Octahedral \\
\hline & 347 & 1.315 & 28818 & 1315 & C.T. & & \\
\hline & 374 & 0.855 & 26737 & 855 & C.T. & & \\
\hline & 502 & 0.207 & 19920 & 207 & ${ }^{4} \mathrm{~T}_{1} \mathrm{~g}(\mathrm{~F}) \rightarrow{ }^{4} \mathrm{~T}_{1} \mathrm{~g}(\mathrm{P})$ & & \\
\hline & 730 & 0.074 & 13698 & 74 & ${ }^{4} \mathrm{~T}_{1} \mathrm{~g}(\mathrm{~F}) \rightarrow{ }^{4} \mathrm{~A}_{2} \mathrm{~g}$ & & \\
\hline & 988 & 0.052 & 101121 & 52 & ${ }^{4} \mathrm{~T}_{1} \mathrm{~g}(\mathrm{~F}) \rightarrow^{4} \mathrm{~T}_{2} \mathrm{~g}(\mathrm{~F})$ & & \\
\hline \multirow{5}{*}[\mathrm{Ni}_{2}(\mathrm{L})(\mathrm{H}_{2}\mathrm{O})_{7}]{$\cdot \mathrm{H}_{2} \mathrm{O}$} & 275 & 1.761 & 36363 & 1761 & L-F. & 2.89 & Octahedral \\
\hline & 375 & 0.546 & 26666 & 546 & C.T. & & \\
\hline & 575 & 0.112 & 17482 & 112 & ${ }^{3} \mathrm{~A}_{2} \mathrm{~g} \rightarrow{ }^{3} \mathrm{~T}_{1} \mathrm{~g}(\mathrm{P})$ & & \\
\hline & 750 & 0.063 & 13333 & 63 & ${ }^{3} \mathrm{~A}_{2} \mathrm{~g} \rightarrow{ }^{3} \mathrm{~T}_{1} \mathrm{~g}(\mathrm{~F})$ & & \\
\hline & 870 & 0.033 & 11494 & 33 & ${ }^{3} \mathrm{~A}_{2} \mathrm{~g} \rightarrow{ }^{3} \mathrm{~T}_{2} \mathrm{~g}(\mathrm{~F})$ & & \\
\hline \multirow[t]{3}{*}[\mathrm{Cu}_{2}(\mathrm{L})(\mathrm{H}_{2}\mathrm{O})_{7}]{$\cdot \mathrm{H}_{2} \mathrm{O}$} & 279 & 1.770 & 34842 & 1770 & L-F. & 1.73 & Octahedral \\
\hline & 370 & 0.553 & 27027 & 553 & C.T. & & \\
\hline & 827 & 0.025 & 12091 & 25 & ${ }^{2} \mathrm{Eg} \rightarrow{ }^{2} \mathrm{~T}_{2} \mathrm{~g}$ & & \\
\hline \multirow[t]{2}{*}[\mathrm{Zn}_{2}(\mathrm{L})(\mathrm{H}_{2}\mathrm{O})_{7}]{$\cdot \mathrm{H}_{2} \mathrm{O}$} & 277 & 1.430 & 36101 & 1430 & L.F. & Dia & Octahedral \\
\hline & 372 & 0.355 & 26881 & 355 & C.T. & & \\
\hline \multirow[t]{2}{*}[\mathrm{Cd}_{2}(\mathrm{L})(\mathrm{H}_{2}\mathrm{O})_{3}]{$\cdot \mathrm{H}_{2} \mathrm{O}$} & 277 & 1.430 & 36101 & 1430 & L-F. & Dia & Tetrahedral \\
\hline & 372 & 0.355 & 26881 & 355 & C.T. & & \\
\hline \multirow[t]{2}{*}[\mathrm{Hg}(\mathrm{L})(\mathrm{H}_{2}\mathrm{O})]{$\cdot \mathrm{H}_{2} \mathrm{O}$} & 271 & 1.635 & 36900 & 1635 & L-F. & Dia & Tetrahedral \\
\hline & 380 & 0.488 & 26315 & 488 & C.T. & & \\
\hline
\end{tabular}


the spectrum shows peaks at 279 and $342 \mathrm{~nm}$ lead to $(\pi-$ $\left.\pi^{*}\right)$ and $\left(n-\pi^{*}\right)$ electronic transition [26]. The spectrum of $\mathrm{Cr}^{3+}$ complex display two peaks at 276 and $364 \mathrm{~nm}$ described to ligand felid and charge transfer respectively, other three peaks at 449, 552 and $670 \mathrm{~nm}$ which were assigned to electronic transition type ${ }^{4} \mathrm{~A}_{2} \mathrm{~g} \rightarrow{ }^{4} \mathrm{~T}_{1} \mathrm{~g}(\mathrm{p}),{ }^{4} \mathrm{~A}_{2} \mathrm{~g}$ $\rightarrow{ }^{4} \mathrm{~T}_{1} \mathrm{~g}(\mathrm{~F})$ and ${ }^{4} \mathrm{~A}_{2} \mathrm{~g} \rightarrow{ }^{4} \mathrm{~T}_{2} \mathrm{~g}(\mathrm{~F})$ respectively, also the value of the magnetic moment at 3.66 B.M may be taken as additional evidence for octahedral geometry [27]. The spectrum of $\mathrm{Mn}^{2+}$ complex displays three peaks at 277 , 347 and $380 \mathrm{~nm}$ due to the ligand field and charge transfer. Peaks at 520 and $809 \mathrm{~nm}$ assigned to electronic transition type ${ }^{6} \mathrm{~A}_{1} \mathrm{~g} \rightarrow{ }^{4} \mathrm{~A}_{1} \mathrm{~g},{ }^{4} \mathrm{Eg}(\mathrm{G}),{ }^{6} \mathrm{~A}_{1} \mathrm{~g} \rightarrow{ }^{4} \mathrm{~T}_{2} \mathrm{~g}(\mathrm{G})$ and ${ }^{6} \mathrm{~A}_{1} \mathrm{~g} \rightarrow{ }^{4} \mathrm{~T}_{1} \mathrm{~g}(\mathrm{G})$ respectively, the magnetic moment of this complex was found at 6.05 B.M which was so close for the octahedral environment [28-29]. The spectrum of $\mathrm{Co}^{2+}$ complex appears three peaks at 276, 347 and $374 \mathrm{~nm}$ which were described to ligand field and charge transfer, peaks at 502, 730 and $988 \mathrm{~nm}$ due to electronic transition type ${ }^{4} \mathrm{~T}_{1} \mathrm{~g}(\mathrm{~F}) \rightarrow{ }^{4} \mathrm{~T}_{1} \mathrm{~g}(\mathrm{P}),{ }^{4} \mathrm{~T}_{1} \mathrm{~g}(\mathrm{~F}) \rightarrow{ }^{4} \mathrm{~A}_{2} \mathrm{~g}$ and ${ }^{4} \mathrm{~T}_{1} \mathrm{~g}(\mathrm{~F}) \rightarrow$ ${ }^{4} \mathrm{~T}_{2} \mathrm{~g}(\mathrm{~F})$ respectively, also the value of the magnetic moment at 5.25 B.M may be taken as additional evidence for octahedral geometry [30]. The $\mathrm{Ni}^{2+}$ complex exhibited two absorption peaks at 275 and $375 \mathrm{~nm}$ due to the ligand field and charge transfer. The other three peaks at 572, 750 and $870 \mathrm{~nm}$ have been appointed in to electronic transition type ${ }^{3} \mathrm{~A}_{2} \mathrm{~g} \rightarrow{ }^{3} \mathrm{~T}_{1} \mathrm{~g}(\mathrm{P}),{ }^{3} \mathrm{~A}_{2} \mathrm{~g} \rightarrow{ }^{3} \mathrm{~T}_{1} \mathrm{~g}(\mathrm{~F})$ and ${ }^{3} \mathrm{~A}_{2} \mathrm{~g} \rightarrow$ ${ }^{3} \mathrm{~T}_{2} \mathrm{~g}(\mathrm{~F})$ respectively. The magnetic moment for this compound was found in 2.89 B.M which was very close for octahedral geometry [31]. The $\mathrm{Cu}^{2+}$ complex appears two peaks at 279 and $370 \mathrm{~nm}$ due to the ligand field as well charge transfer, whilst the third peak at $827 \mathrm{~nm}$ described to electronic transition type ${ }^{2} \mathrm{Eg} \rightarrow{ }^{2} \mathrm{~T}_{2} \mathrm{~g}$, the magnetic moment for this compound was found at 1.73 B.M which was octahedral geometry [32]. Electronic spectra for $\mathrm{Zn}^{2+}, \mathrm{Cd}^{2+}$ and $\mathrm{Hg}^{2+}$ compounds do display the charge transfer, as well the magnetic susceptibility offers that three compounds have diamagnetic moments because the $d-d$ transition is not possible subsequently electronic spectra did not give any productive information, at $\mathrm{Zn}^{2+}$ complex this result agrees with the previous work of octahedral geometry but the $\mathrm{Cd}^{2+}$ as well $\mathrm{Hg}^{2+}$ complexes for geometry are tetrahedral [3334].

\section{Infrared Spectra and Mode of Bonding}

FT-IR spectra of Schiff base (L) and the prepared its metal complexes have been compared, and the data was recorded in Table 3 and Fig. 3, 4 for the ligand and its metal complexes, respectively. The FT-IR spectrum of Schiff base exhibited broad bands at (3230-3350) $\mathrm{cm}^{-1}$, which have been appointed to the stretching vibration of

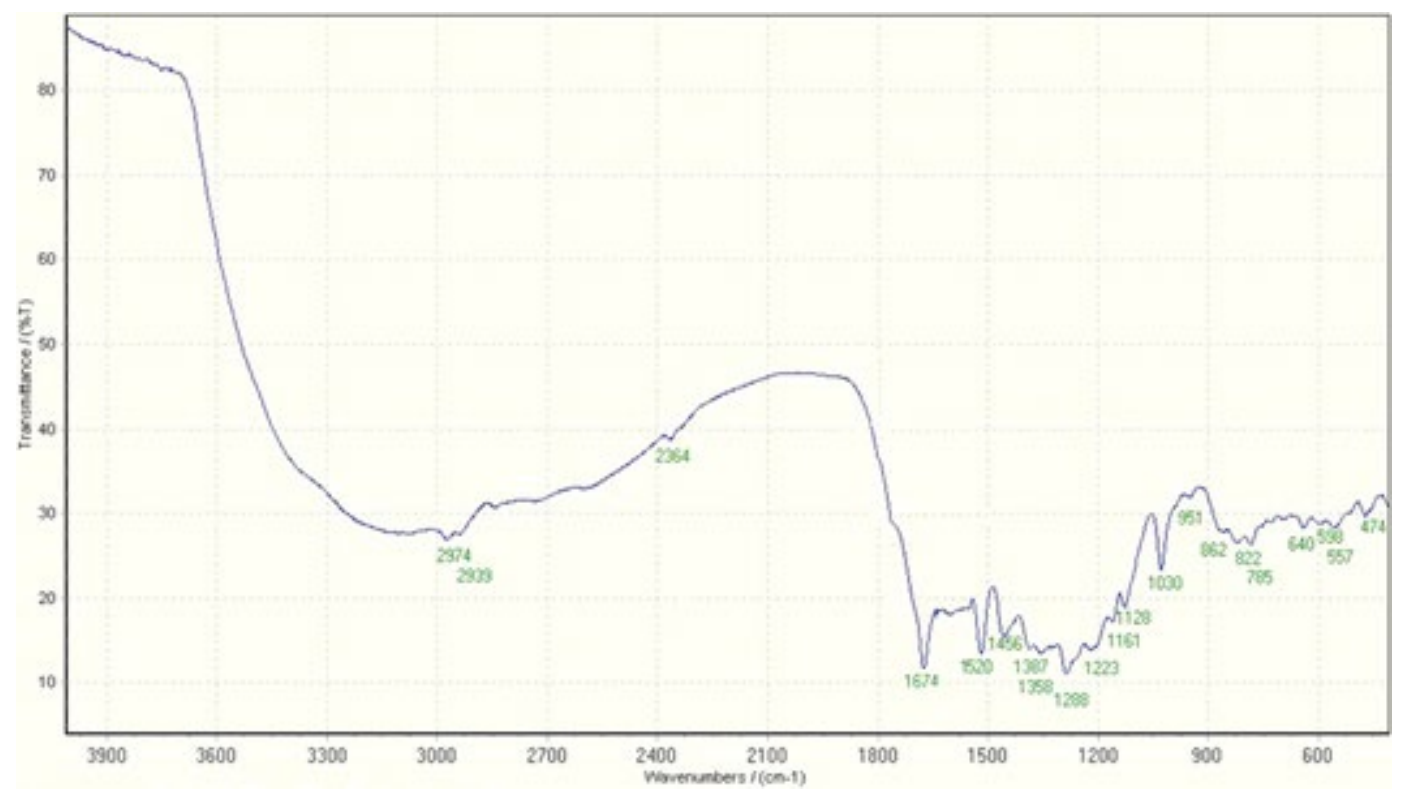

Fig 3. IR spectrum of ligand 


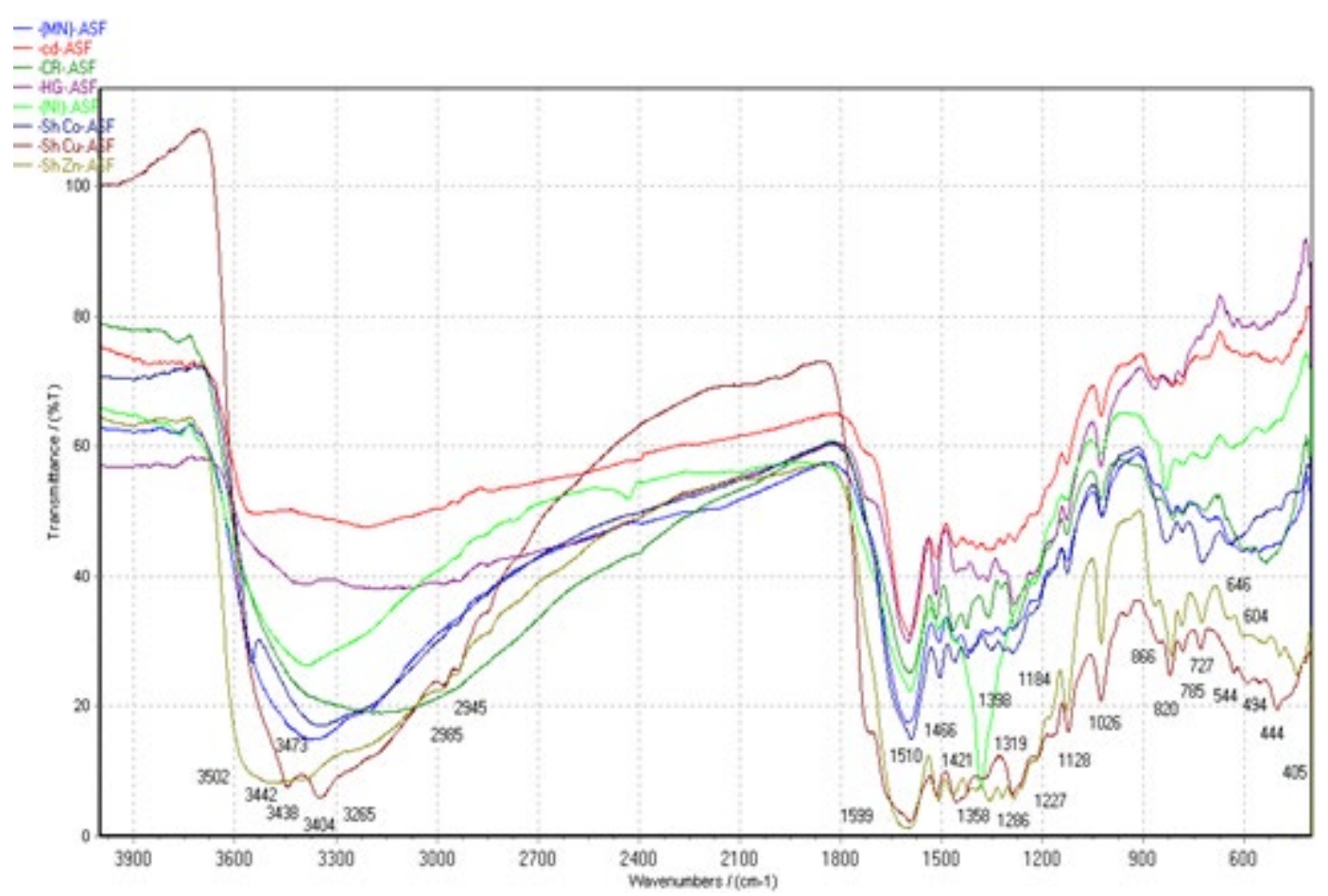

Fig 4. IR spectrum of all metal complexes

Table 3: Selected FT.IR Data (4000-400) $\mathrm{cm}^{-1}$ for Schiff base ligand as well its metal compounds

\begin{tabular}{|c|c|c|c|c|c|c|}
\hline Compounds & $\begin{array}{c}v\left(\mathrm{H}_{2} \mathrm{O}\right) \text { hydrate } \\
\text { and Coord. }\end{array}$ & $v(\mathrm{OH}), v(\mathrm{C}=\mathrm{N})$ & $v_{\text {asy,sy }}\left(\mathrm{COO}^{-}\right)$ & $\delta\left(\mathrm{H}_{2} \mathrm{O}\right)$ & $v(M-N)$ & $v(\mathrm{M}-\mathrm{O})$ \\
\hline Schiff base $(\mathrm{L})$ & - & $3230-3350$ br., $1674 \mathrm{~s}$. & 1520 sh., $1387 \mathrm{~m}$. & - & - & - \\
\hline$\left[\mathrm{Cr}_{2}(\mathrm{~L})\left(\mathrm{H}_{2} \mathrm{O}\right)_{7}\right] \cdot \mathrm{Cl}_{2} \mathrm{H}_{2} \mathrm{O}$ & $\begin{array}{l}3410 \text { br., } \\
3330 \text { br. }\end{array}$ &,$- 1596 \mathrm{~s}$ & 1518 sh., 1361 sh. & $719 \mathrm{w}$. & $602 \mathrm{w}$. & $534 \mathrm{w}$. \\
\hline$\left[\mathrm{Mn}_{2}(\mathrm{~L})\left(\mathrm{H}_{2} \mathrm{O}\right)_{7}\right] \cdot \mathrm{H}_{2} \mathrm{O}$ & $\begin{array}{l}3352 \text { br., } \\
3372 \text { br. }\end{array}$ &,$- 1601 s$ & 1508 sh., 1358 m. & $816 \mathrm{w}$. & $609 \mathrm{w}$. & $496 \mathrm{w}$. \\
\hline$\left[\mathrm{Co}_{2}(\mathrm{~L})\left(\mathrm{H}_{2} \mathrm{O}\right)_{7}\right] \cdot \mathrm{H}_{2} \mathrm{O}$ & $\begin{array}{l}3550 \text { br., } \\
3334 \text { br. }\end{array}$ &,$- 1589 \mathrm{~s}$. & 1504 sh., 1354 m. & $727 \mathrm{w}$. & $494 \mathrm{w}$. & $436 \mathrm{w}$. \\
\hline$\left[\mathrm{Ni}_{2}(\mathrm{~L})\left(\mathrm{H}_{2} \mathrm{O}\right)_{7}\right] \cdot \mathrm{H}_{2} \mathrm{O}$ & $\begin{array}{l}3386 \text { br., } \\
3356 \text { br. }\end{array}$ & -, $1593 \mathrm{~s}$ & 1510 sho, 1381 sh. & $723 \mathrm{w}$. & $544 \mathrm{w}$. & $490 \mathrm{w}$. \\
\hline$\left[\mathrm{Cu}_{2}(\mathrm{~L})\left(\mathrm{H}_{2} \mathrm{O}\right)_{7}\right] \cdot \mathrm{H}_{2} \mathrm{O}$ & $\begin{array}{l}3442 \text { br., } \\
3346 \text { br. }\end{array}$ & -, $1593 \mathrm{~s}$ & 1516 s., $1381 \mathrm{~m}$. & $731 \mathrm{w}$. & $503 \mathrm{w}$. & $465 \mathrm{w}$. \\
\hline$\left[\mathrm{Zn}_{2}(\mathrm{~L})\left(\mathrm{H}_{2} \mathrm{O}\right)_{7}\right] \cdot \mathrm{H}_{2} \mathrm{O}$ & $\begin{array}{l}3504 \text { br., } \\
3400 \text { br. }\end{array}$ &,$- 1597 \mathrm{~s}$. & 1508 sh., $1358 \mathrm{~m}$. & $727 \mathrm{w}$. & $523 \mathrm{w}$. & $496 \mathrm{w}$. \\
\hline$\left[\mathrm{Cd}_{2}(\mathrm{~L})\left(\mathrm{H}_{2} \mathrm{O}\right)_{3}\right] \cdot \mathrm{H}_{2} \mathrm{O}$ & $\begin{array}{l}3545 \text { br., } \\
3360 \text { br. }\end{array}$ &,$- 1595 \mathrm{sh}$ & 1516 sh., 1358 m. & $708 \mathrm{w}$. & $521 \mathrm{w}$. & $485 \mathrm{w}$. \\
\hline$\left[\mathrm{Hg}(\mathrm{L})\left(\mathrm{H}_{2} \mathrm{O}\right)\right] \cdot \mathrm{H}_{2} \mathrm{O}$ & $\begin{array}{l}3384 \text { br., } \\
3350 \text { br. }\end{array}$ & 3266 br., 1601 sh. & 1518 sh., 1385 m. & $733 \mathrm{w}$. & $552 \mathrm{w}$. & $486 \mathrm{w}$. \\
\hline
\end{tabular}

br.=broad, sh.-sharp, s.-strong, sho.=shoulder, w.=weak

$v(\mathrm{OH})$ carboxylic and phenol groups, these bands disappear in the all spectra of complexes described to coordinated these groups in coordination [35], except the
$\mathrm{Hg}^{2+}$ complex the coordinated through the $(\mathrm{OH})$ for carboxyl and phenol of $o$-vanillin. Band in $(1674) \mathrm{cm}^{-1}$ whom has been assigned into $v(\mathrm{C}=\mathrm{N})$ group of Schiff 
base ligand, suffered a great change to lower frequency was observed on complexation with a metal ion, lead to coordinated of this group at coordination [36].

The bands at 1520 and $1387 \mathrm{~cm}^{-1}$ have been appointed into stretching vibration for Schiff base ligand of $v\left(\mathrm{COO}^{-}\right)$asymmetric as well symmetric respectively, these bands were shifted into lower frequencies, may result to coordination with metal ions [37]. The presence of water hydrate at the scope $(3352-3550) \mathrm{cm}^{-1}$ and coordinated in the spectra of all complexes were suggested by the very broad absorption bands around (3330-3400) $\mathrm{cm}^{-1}$ and show peaks at $(708-816) \mathrm{cm}^{-1}$ due to stretching, rocking and wagging modes of coordination water molecules [38]. Some new bands' weak intensity observed in the regions around (436-609) $\mathrm{cm}^{-1}$ may be ascribed to $v(\mathrm{M}-\mathrm{N})$ and $v(\mathrm{M}-\mathrm{O})$ vibration respectively [39]. According to the result data the geometry has been suggested at.

\section{Mass Spectra for Schiff Base Ligand as Well Its Metal Compounds}

The mass spectra fragmentation patterns for free Schiff base $(\mathrm{L})$ were in good agreement with the suggested structure in Fig. 5. The mass spectrum was characterized by an intense peak at $(\mathrm{m} / \mathrm{z}=345.37)$, which corresponding $\left[\mathrm{M}^{+}\right]$. The mass spectra of Fig. 6 is for Co complex each

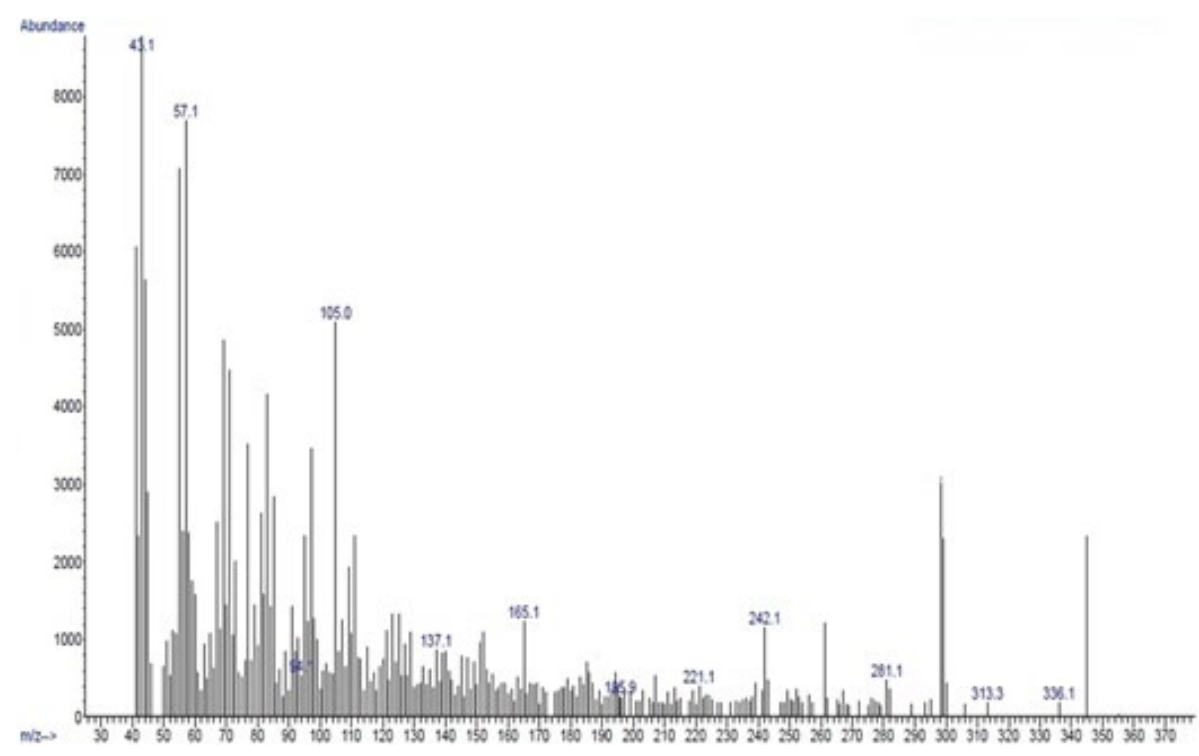

Fig 5. Mass spectral of the Schiff base

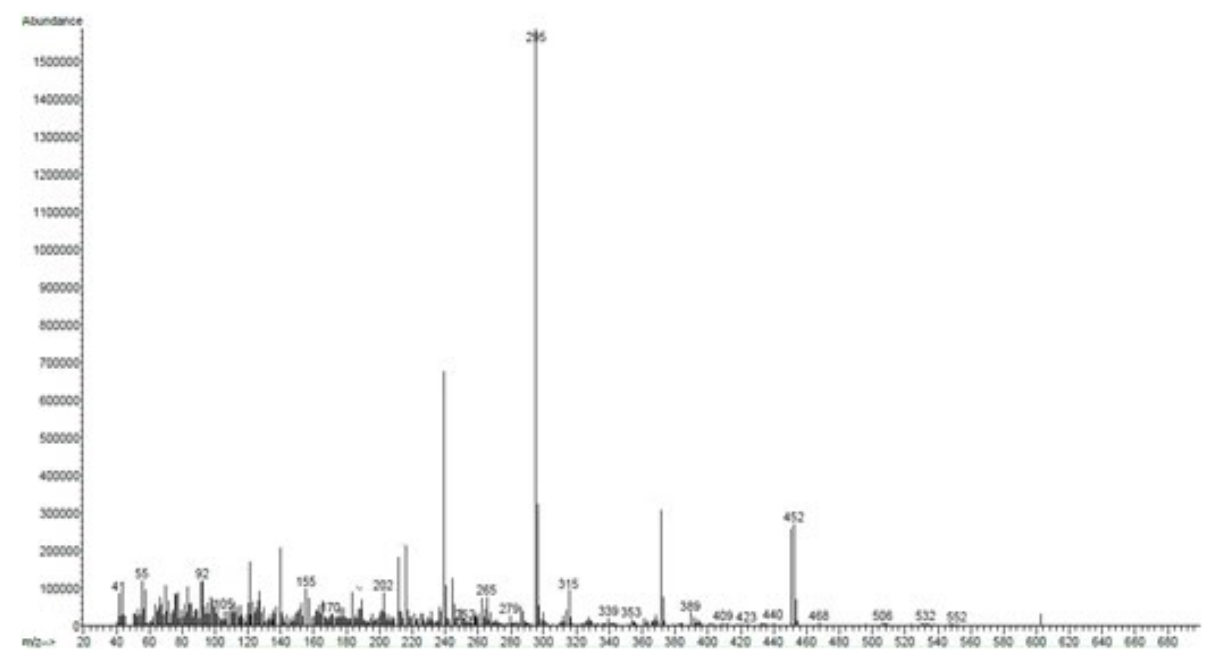

Fig 6. Mass spectral of the Co complex 
spectrum of $\mathrm{Cr}^{3+}, \mathrm{Mn}^{2+}, \mathrm{Co}^{2+}, \mathrm{Ni}^{2+}, \mathrm{Cu}^{2+}, \mathrm{Zn}^{2+}, \mathrm{Cd}^{2+}$ and $\mathrm{Hg}^{2+}$ complexes, respectively. Displayed peaks referred in the molecular ions $\mathrm{m} / \mathrm{z}$ at $660.27,595.33,603.23,602.73$, 612.46, 620.13, 638.19 and $579.96 \mathrm{M}^{+}$into $\mathrm{Cr}^{3+}, \mathrm{Mn}^{2+}$, $\mathrm{Co}^{2+}, \mathrm{Ni}^{2+}, \mathrm{Cu}^{2+}, \mathrm{Zn}^{2+}, \mathrm{Cd}^{2+}$ and $\mathrm{Hg}^{2+}$ complexes, consecutively. That datum is at aood convention for the suggestion of molecular formulation into the complexes.

\section{Antimicrobial Bioassay}

Antibacterial and Antifungal activity of Schiff base ligand and its metal complexes were tested in vitro against bacteria such as Steptococcus epidermidis, Staphylococcus aureus, Klebsiella sp. and Escherichia coli and fungal Candida albicans (yeast) through paper disc plate method
[40]. The compounds have been examined at condensation $10^{-3}$ in DMSO Shown in Table 4, Fig. 7 and Fig. 8. From Table 4, it is clear which the inhibition through metal chelates is higher than that with $\mathrm{Cd}^{2+}$ complex to the against bacteria Steptococcus epidermidis), Staphylococcus aureus, Klebsiella sp., and Escherichia. Coli compared to Schiff base and other complexes and with $\mathrm{Hg}^{2+}$ complex to the versus fungal Candida albicans (yeast).

\section{- CONCLUSION}

On the start of results got after portrayal and antibacterial, antifungal examinations clearly all the blended Schiff base metal complexes showed octahedral

Table 4. Antibacterial and antifungal activities for Schiff base ligand as well its metal compounds in the form of inhibition zone diameter $(\mathrm{mm})$

\begin{tabular}{cccccc}
\hline Compounds & $\begin{array}{c}\text { Steptococcus } \\
\text { epidermidis }(\mathrm{G}+\mathrm{ev})\end{array}$ & $\begin{array}{c}\text { Staphylococcus } \\
\text { aureus }(\mathrm{G}+\mathrm{ev})\end{array}$ & $\begin{array}{c}\text { Klebsiella sp. } \\
\text { (G-ev) }\end{array}$ & $\begin{array}{c}\text { Escherichia. } \\
\text { ColiB(G-ev) }\end{array}$ & $\begin{array}{c}\text { Candida albicans } \\
\text { (Yeast) }\end{array}$ \\
\hline L & 12 & 10 & 10 & 12 & 11 \\
Cr-complex & - & 11 & - & 12 & - \\
Mn-complex & - & 15 & 9 & 9 & - \\
Co-complex & - & 15 & - & 13 & - \\
Ni-complex & - & - & - & 11 & - \\
Cu-complex & - & - & - & - & 18 \\
Zn-complex & 12 & 25 & 14 & 13 & 22 \\
Cd-complex & 20 & 33 & 19 & 19 & 25 \\
Hg-complex & 22 & 30 & 15 & 21 & 29 \\
\hline
\end{tabular}

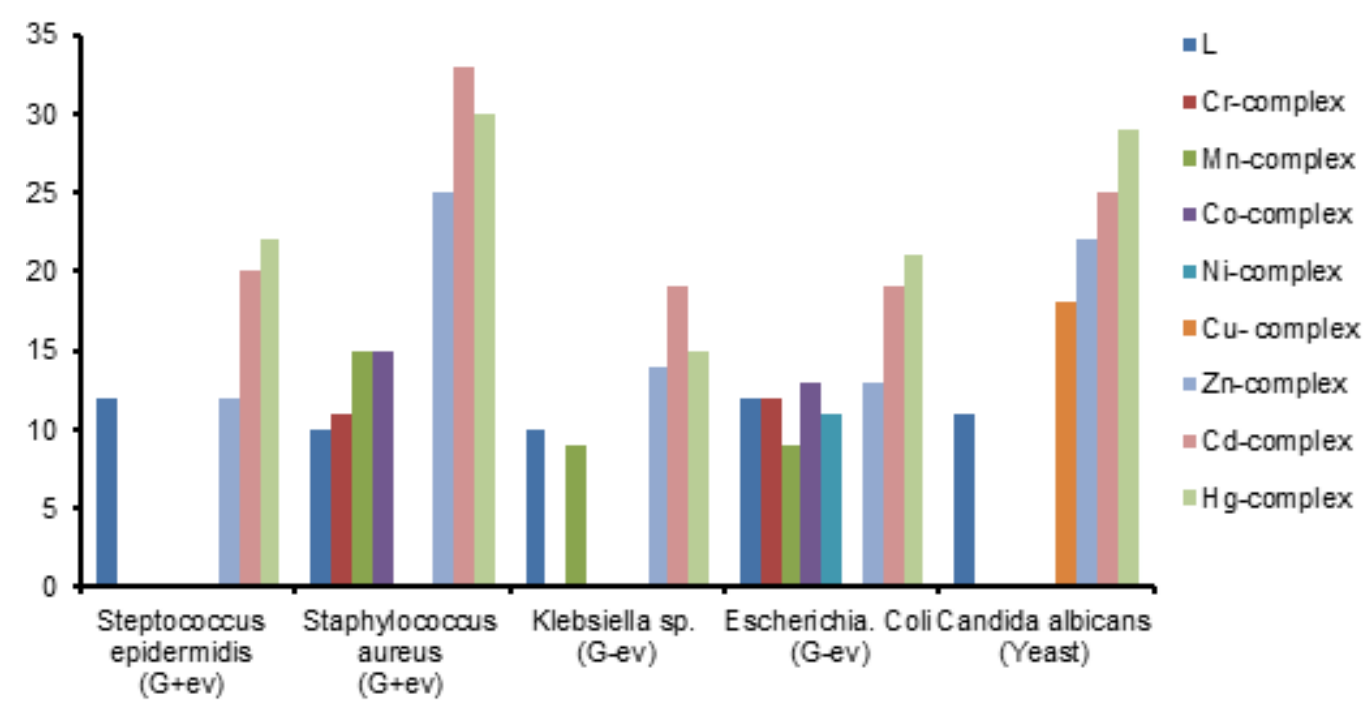

Fig 7. Results of antibacterial and antifungal screening for the Schiff base ligand and its metal compounds 


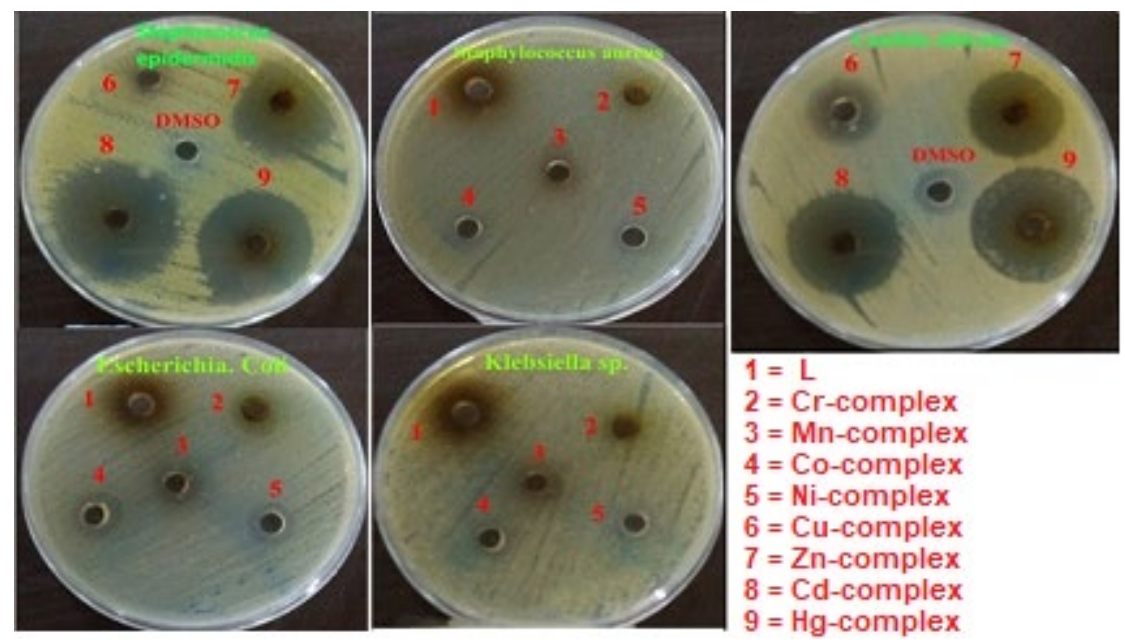

Fig 8. Antibacterial activity of investigated compounds against Steptococcus epidermidis, Staphylococcus aureus, Klebsiella sp. and Escherichia coli and antifungal activity evaluation against Candida albicans (Yeast) for Schiff base and its metal compounds

geometry and upgraded antibacterial, antifungal properties against chose microorganisms. These perceptions, as per diverse examinations, suggest that metal based drugs have potential as therapeutics. These preliminary outcomes, got from in vitro tries, might be enhanced by other more comprehensive studies in vivo, both in controlled conditions and in an open field for all intents and purposes assess the utilization of these complexes on the edge of biological applications.

\section{- REFERENCES}

[1] Yousif, E., Majeed, A., Al-Sammarrae, K.., Salih, N., Salimon, J., and Abdullah, B., 2017, Metal complexes of Schiff base: Preparation, characterization and antibacterial activity, Arabian J. Chem., 10 (Suppl. 2), S1639-S1644.

[2] Sarwar, A., Shamsuddin, M.B., and Lingtang, H., 2018, Synthesis, characterization and luminescence studies of metal-diimine complexes, Mod. Chem. Appl., 6 (3), 1-7.

[3] Iniama, G.E., Iorkpiligh, I.T., and Alfred, A.I., 2018, Stereochemical characterization and antimicrobial activities of synthesized $\mathrm{Ni}(\mathrm{II}), \mathrm{Cu}(\mathrm{II})$ and $\mathrm{Zn}$ (II) Schiff base complexes derived from $p$-nitroaniline and salicylaldehyde, IJST, 6 (5), 52-56.

[4] Divya, K., Pinto, G.M., and Pint, A.F., 2017, Application of metal complexes of Schiff bases as an antimicrobial drug: A review of recent works, Int. J. Curr. Pharm. Res., 9 (3), 27-30.

[5] Al Zoubi, W., Al-Hamdani, A.A.S., and Ko, Y.G., 2017, Schiff bases and their complexes: Recent progress in thermal analysis, Sep. Sci. Technol., 52 (6), 1052-1069.

[6] Şabik, A.E., Karabörk, M., Ceyhan, G., Tümer, M., and Diğrak, M., 2012, Polydentate Schiff base ligands and their $\mathrm{La}(\mathrm{III})$ complexes: Synthesis, characterization, antibacterial, thermal, and electrochemical properties, Int. J. Inorg. Chem., 2012, 791219.

[7] Tobriya, S.K., 2014, Biological applications of Schiff base and its metal complexes-A review, Int. J. Sci. Res., 3 (9), 1254-1256.

[8] Prakash, A., and Ahmad, S., 2018, Synthesis and characterization of Schiff base complexes with $\mathrm{Ti}(\mathrm{III}), \mathrm{Cr}(\mathrm{III})$ and $\mathrm{Ni}(\mathrm{II})$, Orient. J. Chem., 25 (4), 1035-1040.

[9] Al Zoubi, W., Al-Hamdani, A.A.S., Ahmed, S.D., and Ko, Y.G., 2018, Synthesis, characterization, and biological activity of Schiff bases metal complexes, J. Phys. Org. Chem., 31 (3752), 1-13.

[10] Al-Hamdani, A.A.S., and Al Zoubi, W., 2015, New metal complexes of $\mathrm{N}_{3}$ tridentate ligand: Synthesis, spectral studies, and biological activity, Spectrochim. Acta, Part A, 137, 75-89. 
[11] Al-Hamdani, A.A.S., Al-Dulyme, N.K.G., Ahmed, S.D., and Basheer, H.M., 2017, Preparation, spectroscopic, bioactive and theoretical studies of mixed ligand complexes, Al-Nahrain J. Sci., 20 (3), 49-59.

[12] Malik, A., Goyat, G., Vikas, K., Verma, K.K., and Garg, S., 2018, Coordination of tellurium(IV) with Schiff base derived from $o$-vanillin and 3aminopyridine, Int. J. Chem. Sci., 6 (1), 1-10.

[13] Ejidike, I.P., and Ajibade, P.A., 2015, Transition metal complexes of symmetrical and asymmetrical Schiff bases as antibacterial, antifungal, antioxidant and anticancer agents: Progress and prospects, Rev. Inorg. Chem., 35 (4), 191-224.

[14] Borase, J.N., Mahale, R.G., and Rajput, S.S., 2017, Design, synthesis, and biological evaluation of a novel class of heterocyclic Schiff bases, Eur. J. Biomed. Pharm. Sci., 4 (10), 842-845.

[15] Neelakantan, M.A., Esakkiammal, M., Mariappan, S.S., Dharmaraja, J., and Jeyakumarit, A., 2010, Synthesis, characterization and biocidal activities of some Schiff base metal complexes, Indian J. Pharm. Sci., 72 (2), 216-222.

[16] Begum, N.T., Raju, J.A., Nageswara, R.G., and Sreeramulu, J., 2014, Spectroscopic characterization and biological evolution of ortho vanillin pramipexole Schiff base metal complexes, Der Pharma Chem., 6 (2), 51-58.

[17] World Health Organization, 2013, WHO Model List of Essential Medicines, $18^{\text {th }}$ list, October 2013, https://www.who.int/medicines/publications/essenti almedicines/en/, accessed on 22 April 2014.

[18] Jain, S., Jain, N.K., and Pitre, K.S., 2002, Electrochemical analysis of sparfloxacin in pharmaceutical formulation and biochemical screening of its Co(II) complex, J. Pharm. Biomed. Anal., 29 (5), 795-801.

[19] Chandra, S., Shukla, D., and Gupta, L.K., 2008, Synthesis and spectroscopic studies of cobalt(II), nickel(II), and copper(II) complexes with N-donor (N4) macrocyclic ligand (DSLF), J. Indian Chem. Soc., 85 (5), 800-806.
[20] Vogel, A.I.., 1978, Text Book of Quantitative Inorganic Analysis, $4^{\text {th }}$ Ed., Longman, London, 694.

[21] Geary, W.J., 1971, The use of conductivity measurements in inorganic solvents for the characterization of coordination compounds, Coord. Chem. Rev., 7, 81-122.

[22] Singh, B.K., Rajour, H.K., and Prakash, A., 2012, Synthesis, characterization and biological activity of transition metal complexes with Schiff bases derived from 2-nitrobenzaldehyde with glycine and methionine, Spectrochim. Acta, Part A, 94, 143-151.

[23] Silverstein, R.M., Webster, X.F., and Kiemle, D.J., 2005, Spectrometric Identification of Organic Compounds, $7^{\text {th }}$ Ed., John Wiley \& Son, Inc., Hoboken, New York.

[24] Khaleel, A.M.N., and Jaafar, M.I., 2017, Synthesis and characterization of boron and 2-aminophenol Schiff base ligands with their $\mathrm{Cu}(\mathrm{II})$ and $\mathrm{Pt}(\mathrm{IV})$ complexes and evaluation as antimicrobial agents, Orient. J. Chem., 33 (5), 2394-2404.

[25] Al-Hamdani, A.A.S., Ahmed, S.D., Shake, S.H., and Hassan, Z.A., 2016, Synthesis and spectroscopic characterization for some metal ion complexes with 2-hydroxy-3-((5-mercapto-1,3,4-thiadiazol-2-yl)dia zenyl)-1-naphthaldehyde, Baghdad Sci. J., 13, 105114.

[26] Al Zoubi, W., Al-Hamdani, A.A.S., Widiantara, I.P., Hamoodah, R.G., and Ko, Y.G., 2017, Theoretical studies and antibacterial activity for Schiff base complexes, J. Phys. Org. Chem., 30 (12), e3707.

[27] Lever, A.B.P., 1968, Inorganic Electronic Spectroscopy, Elsevier, Amsterdam, 121.

[28] Ghanim, F.H., 2016, Synthesis, characterization and antibacterial activities of $\mathrm{Mn}$ (II), $\mathrm{Fe}(\mathrm{II}), \mathrm{Co}(\mathrm{II})$, $\mathrm{Ni}(\mathrm{II}), \mathrm{Cu}(\mathrm{II}), \mathrm{Zn}(\mathrm{II})$ and $\mathrm{Cd}(\mathrm{II})$ mixed-ligand complexes of 8-hydroxyquinoline and sulfamethoxazole, Trans. Eng. Sci., 4 (2), 73-76.

[29] Shaker, S.A., Mohammed, H.A., and Al-Hamdani, A.A.S., 2010, Preparation, physico-chemical and spectroscopic investigation of thiacetazone and quinalizarin complexes with $\mathrm{Mn}(\mathrm{II}), \mathrm{Fe}(\mathrm{II}), \mathrm{Co}(\mathrm{II})$, 
$\mathrm{Ni}(\mathrm{II}), \mathrm{Cu}(\mathrm{II}), \mathrm{Zn}(\mathrm{II}), \mathrm{Cd}(\mathrm{II})$ and $\mathrm{Pb}(\mathrm{II})$, Aus. J. Basic Appl. Sci., 4 (10), 5178-5183.

[30] Raja, R., Verma, K.K., Solanki, K., and Bhojak, N., 2016, Synthesis, characterization and antimicrobial activity of cobalt(II) complexes with pyrimidine derivatives, Res. J. Chem. Sci., 6 (3), 48-55.

[31] AL-Hamdani, A.A.S., and Shaker, S.A., 2011, Synthesis, characterization, structural studies, and biological activity of a new Schiff base- azo ligand and its complexation with selected metal ions, Orient. J. Chem., 27 (3), 835-845.

[32] AL-Hamdani, A.A.S., Balkhi, A.M., Falah, A., and Shaker, S.A., 2015, New azo-Schiff base derived with $\mathrm{Ni}(\mathrm{II}), \mathrm{Co}(\mathrm{II}), \mathrm{Cu}(\mathrm{II}), \mathrm{Pd}(\mathrm{II})$ and $\mathrm{Pt}(\mathrm{IV})$ complexes: Preparation, spectroscopic investigation, structural studies, and biological activity, J. Chil. Chem. Soc., 60 (1), 2774-2785.

[33] Al-Noor, T.H., Jarad, A.J., and Abo, S.B., 2015, Synthesis, spectral and antimicrobial activity of mixed ligand complexes of $\mathrm{Co}(\mathrm{II}), \mathrm{Ni}(\mathrm{II}), \mathrm{Cu}(\mathrm{II})$ and $\mathrm{Zn}(\mathrm{II}) \quad$ with 4-aminoantipyrine and tributylphosphine, Int. J. Curr. Res., 7 (5), 1560515609.

[34] Obaid, S.M.H., Al Naemi, H.A.S., Jarad, A.J., AlHamdani, A.A.S., and Fendi, W.J., 2017, Preparation, spectral studies and biological efficiency of metal (II) mixed ligand complexes Schiff base derived from (benzaldehyde and $o$-aminoaniline) with 8hydroxyquinoline, Res. J. Pharm. Biol. Chem. Sci., 8 (6), 179-189.
[35] Nakamoto, K., 1997, Infrared and Raman Spectra of Inorganic and Coordination Compounds, Applications in Coordination, Organometallic, and Bioinorganic Chemistry, $5^{\text {th }}$ Ed., Wiley-Inter Science, New York.

[36] Silverstein, R.M., Bassler, G.C., and Morril, T.C., 1981, Spectroscopic Identification of Organic Compounds, $4^{\text {th }}$ Ed., Wiley, New York.

[37] Obaid, S.M.H., Al Naemi, H.A.S., and Al-Hamdani, A.A.S., 2018, Synthesis, spectroscopic and antimicrobial studies of mixed ligand metal(II) complexes with three amino acids, Res. J. Pharm. Biol. Chem. Sci., 9 (6), 1560-1571.

[38] Al Zoubi, W., Al-Hamdani, A.A.S., Ahmed, S.D., and Ko, Y.G., 2018, A new azo-Schiff base: Synthesis, characterization, biological activity and theoretical studies of its complexes, Appl. Organomet. Chem., 32 (1), e3895.

[39] Abdulghani, A.J., and Hussain, R.K., 2018, Synthesis and characterization of Schiff base metal complexes derived from cefotaxime with $1 \mathrm{H}$ indole-2,3-dione (Isatin) and 4-N,N-dimethylaminobenzaldehyde, Open J. Inorg. Chem., 5 (4), 83-101.

[40] Al-Hamdani, A.A.S., Altayy, M.A.M., and AlDulyme, N.K.G., 2017, Synthesis, spectroscopic and theoretical studies of some new transition metal complexes with mixed ligands Schiff base and bipyridyl, Ibn Al-Haitham J. Pure Appl. Sci., 30 (2), 69-83. 